\title{
TEORÍAS ECONÓMICAS DE LA INTERNACIONALIZACIÓN: EL CONTEXTO EPISTEMOLÓGICO DE LA EXPANSIÓN EMPRESARIAL
}

Economic theories on internationalization: epistemological context of business expansion

María Gabriela Ramos-Barrera

mgramos@poligran.edu.co

Institución Universitaria Politécnico Grancolombiano

Colombia

\section{Resumen}

Los procesos globalizadores y de integración económica han cambiado la forma en la que interactúan las empresas, especialmente por los procesos de internacionalización frente a los que se encuentran expuestas. Por ello, el estudio de la economía internacional es primordial para entender la dinámica de la expansión empresarial, razón por la cual se han establecido diversas teorías que tratan de explicar la internacionalización. Así, este artículo describe cómo la mayor parte de las teorías relacionadas con la economía internacional se han tratado de explicar desde una perspectiva positivista que toma a los modelos como marco de estudio principal y generalizable, tomando en cuenta -también- cómo las teorías más recientes han considerado al interpretativismo y al constructivismo como paradigmas igualmente válidos para la explicación de la internacionalización empresarial. 


\section{INTRODUCCIÓN}

En los procesos de investigación una de las controversias comunes se da en el campo de la epistemología, cuando se trata de definir la interpretación filosófica del conocimiento y la aplicación de este. La economía como ciencia, y la economía internacional como campo de estudio, no se escapan de esta realidad y también discuten sobre la pertinencia del conocimiento generado en torno al enfoque y la metodología que se abarca dentro del desarrollo de las investigaciones.

La creación del conocimiento en economía se ha caracterizado por el uso de métodos cuantitativos, que infieren los resultados de una muestra para una población y permiten la clasificación de las mediciones y las segmentaciones de formas comparables a través del tiempo, pero no permiten hacer análisis profundos sobre el comportamiento de los individuos estudiados dada la inflexibilidad de las técnicas utilizadas.

En principio, la internacionalización se entendía como un fenómeno que respondía netamente a la dotación factorial de los países, medida a través de instrumentos cuantitativos. Con el tiempo, la búsqueda de oportunidades como respuesta a las fallas de los mercados nacionales (como las economías de escala, externalidades, entre otras), definieron los términos del intercambio internacional, pero la perspectiva teórica bajo la cual se definían estas oportunidades comenzó a fortalecerse con enfoques epistemológicos interpretativos y constructivistas.

Por ello, con base en el concepto de ciencia que resalta la estructura sistemática que permite la comprobación experimental, los procesos de internacionalización estarían determinados por estructuras pertenecientes a un sistema internacional preestablecido y organizado, justificando la prevalencia del paradigma positivista dentro de la creación del conocimiento empresarial. Sin embargo, también es cierto que los procesos de expansión comercial se han generado como respuesta a la búsqueda de nuevos mercados, nuevas realidades y adaptaciones tecnológicas que han cambiado los cimientos económicos porque no pueden forjarse sobre el principio del ceteris paribus, pues las variables que fueron consideradas fundamentales en una época han cambiado con el tiempo. Como plantea Gómez (200, p. 71):

El reconocimiento de esta situación ha dado lugar a posturas diferenciadas con importantes consecuencias sobre la definición del status epistemológico de las leyes sociales. Se parte del reconocimiento de que las leyes sociales no pueden considerarse leyes universales en sentido estricto, son leyes cuasigenerales que, aunque son expresadas de forma estrictamente universal, de hecho presentan diversas excepciones.

Con base en estos elementos centrales, el presente artículo de reflexión pretende describir las principales teorías de internacionalización bajo la perspectiva económica, y enlazarlas con tres paradigmas derivados de diferentes enfoques epistemológicos: el positivismo, el interpretativismo y el constructivismo; con el fin de comprender la evolución del conocimiento dentro de la economía internacional y su impacto sobre los estudios empresariales ${ }^{1}$.

Para ello: en primer lugar, se contextualiza el problema de la teorización económica; en segundo lugar, se explica la evolución de las teorías de la internacionalización bajo la perspectiva económica; en tercer lugar, se relacionan los fundamentos epistemológicos de cada teoría y; finalmente, se redactan las principales conclusiones del estudio.

\footnotetext{
${ }^{1} \mathrm{Al}$ respecto vale la pena destacar que, si bien existen teorías que explican la internacionalización empresarial desde el punto de vista del proceso -que considera a esta etapa como una consecuencia directa de la acumulación de conocimientos y el incremento de recursos-, y otras teorías, como las de redes -en donde la internacionalización se explica como un desarrollo lógico y social de las empresas-; en este artículo el contexto epistemológico se limita a la perspectiva económica que explica la internacionalización como una estrategia para corregir las imperfecciones del mercado y disminuir los costos empresariales. Lo anterior, dada la importancia histórica de la perspectiva económica que luego daría paso a las demás teorías.
} 
Una ciencia es entendida como un "conjunto de conocimientos obtenidos mediante la observación y el razonamiento, sistemáticamente estructurados, y de los que se deducen principios y leyes generales con capacidad predictiva y comprobables experimentalmente" (RAE, 2019).

En este orden de ideas, se puede encontrar una ciencia exacta como la matemática, en donde componentes como la construcción de axiomas y el análisis lógico permiten estudiar un contexto abstracto, haciendo que la rigurosidad de las deducciones matemáticas se fundamente en la pureza del método científico utilizado para comprobar la hipótesis planteada. Ahora bien, la Economía como ciencia social estudia al ser humano y su interacción dentro de la sociedad implicando que, dada la complejidad del objeto de estudio, la experimentación del método científico no sea fácil de aplicar y no exista control sobre las variables (o al menos sea poco probable).

Lo anterior ha generado que numerosos economistas solventen la limitación del análisis económico con el uso de las matemáticas, aproximando el método del análisis social a un método basado en una ciencia exacta. Por ejemplo: León Walras, usó un sistema de ecuaciones para explicar el equilibrio estático y ecuaciones de circulación para predecir la demanda del dinero y Kenneth Arrow, hizo un aporte fundamental a la economía con el estudio de funciones de producción de elasticidad constante de sustitución (CES) y el cálculo de la aversión al riesgo (Ramos y Bello, 2020). Así, para algunos economistas, cuando el análisis económico no se basa en información empírica este análisis es especulativo y, por ende, deficiente. Por lo tanto, en esta simbiosis se centra una de las discusiones epistemológicas de la teoría económica, tomando como método inicial para construir el conocimiento al paradigma positivista.

Este paradigma ha permitido que se establezca, sobre la llamada Economía Positiva, una relación causa-efecto entre variables económicas sin considerar juicios de valor. En otras palabras, se pueden construir modelos que reflejen lo que es y no lo que debería ser (Economía Normativa), que le dan prioridad a la razón del por qué surgen ciertos fenómenos con base en una explicación objetiva, soportada en el método científico, y que se limita a la descripción antes que a la comprensión del impacto de una variable económica sobre la dinámica de la sociedad.

Ahora, la comprensión normativa sobre la cual reposan los diseños de la Economía Política tiende a ser sesgada de acuerdo con la percepción de quién formula los instrumentos. Entonces, dada la influencia de la ideología sobre las teorías económicas, otro factor pertinente es que la percepción sobre la cual se construye la realidad será altamente polémica, o como exponen Borgucci y Castellano (2016, p. 29):

En Economía un paradigma legitimado, no significa que sea hegemónico ni siquiera en los problemas y métodos en que se considera fuerte. En Economía para un mismo problema hay diferentes posturas que polemizan, porque responden generalmente a posiciones filosóficas o corrientes ideológicas. Esto nos lleva a la idea de que el paradigma más "popular" convive con posturas críticas.

Empero, la construcción del conocimiento en Economía es un proceso diacrónico, fundamentado sobre la evolución de los fenómenos y la retroalimentación del entendimiento, que comprende el análisis del todo y de las partes. Una de estas partes es la economía internacional que estudia el desempeño comercial de un país, la interacción financiera de este con el resto del mundo, y las alianzas que se generan a nivel mundial.

Desde el Siglo I con la ruta de la seda, el siglo $\mathrm{V}$ con la invención de la moneda, las cruzadas desarrolladas entre los siglos XI y XII, las primeras rutas comerciales y las ferias del siglo XIV, se crearon escenarios bajo los cuales se promovía el intercambio de bienes entre países. Dado el interés por generar mayor riqueza con base en el proceso mercantil, el intercambio comercial comenzó a enfocarse en el oro, las piedras preciosas y las especias, ocasionando el inicio formal del comercio internacional en el siglo XVI. Sin embargo, para De la Hoz (2013:15) "no fue hasta la Revolución Industrial con todas las innovaciones en maquinaria, formas de producción y, por 
consiguiente, en el cambio de estilo de vida que se logró obtener un efecto y trascendencia continental y mundial".

Posteriormente, como consecuencia de las guerras mundiales y la depresión económica de los años 30, el siglo XX se caracterizó por las políticas comerciales proteccionistas con un rol significativo del Estado dentro del intercambio comercial (Sastre, 2014). Pero a partir de 1950, el comercio inició su etapa de crecimiento y auge, caracterizado por el intercambio de los países industrializados y el crecimiento de las inversiones extranjeras. Desde entonces, durante las últimas décadas el comercio internacional ha sufrido cambios estructurales como consecuencia de la revolución tecnológica y el crecimiento del sector de servicios, soportados en un creciente interés por integrar economías y fomentar las ventajas de los procesos globalizadores (Sastre, 2014).

De lo anterior vale la pena destacar que la evolución del comercio internacional, como solución a la satisfacción de las necesidades de consumo, se generó a través del intercambio de importaciones y exportaciones; mientras que el comercio internacional enfocado en las ganancias del intercambio creó un espacio adicional de beneficio al instaurar establecimientos comerciales fuera de las fronteras de su país origen (oficinas de representación), para luego constituirse en productores de bienes y servicios en el extranjero (inversión extranjera) y, finalmente, producir a través de las cadenas globales de valor (empresas globalizadas).

Entonces, acorde con las características evolutivas del comercio internacional, las empresas se internacionalizan por dos razones fundamentales: para satisfacer las necesidades de consumo y para generar beneficios durante el proceso mercantil. Lo anterior ha sido producto de un proceso de aprendizaje en el cual los países adaptaron sus comportamientos hacia el interés individual para maximizar sus beneficios. Este proceso implica una discusión filosófica: la diferencia entre una percepción que se pretende medir con la metodología positivista y el análisis conductista en donde los comportamientos se explican con la relación estímulo-respuesta. El debate epistemológico se centra, entonces, en la forma bajo la cual se entienden los procesos de expansión e internacionalización comercial de los países.

La evolución de las teorías de internacionalización bajo la perspectiva económica: factores de producción e imperfecciones de mercado

El proceso de internacionalización de la producción ha sido estudiado desde el siglo XV cuando Jean Baptiste Colbert, en 1661, desarrolló políticas para dar independencia económica a Francia a través del comercio internacional y una balanza en cuenta corriente superavitaria, fortaleciendo el sistema de transporte terrestre y creando nuevos puertos y rutas comerciales para el transporte marítimo.

Sin embargo, los primeros postulados formales de la internacionalización se encuentran en los aportes de la escuela clásica de economía: para el clásico Adam Smith, los mercantilistas fundamentaron el crecimiento económico en el intercambio comercial de metales preciosos porque consideraban que un país era rico por la abundancia de oro y plata, pero no diferenciaron entre riqueza y acumulación.

Según Smith (1776), la riqueza de una nación dependía exclusivamente del valor del trabajo, dado que el trabajo no es homogéneo. Por lo tanto, si el trabajo es el único factor de producción y los bienes se intercambian de acuerdo con los requerimientos de trabajo necesarios para producirlos, un país puede ser más eficiente que otro en la producción de un bien y menos eficiente que otro en la producción de otro bien. Smith define esta condición como ventaja absoluta, a partir de esta noción se presume que el comercio entre naciones es de muto beneficio. Ahora, para David Ricardo (1817), también representante de la escuela clásica de la economía, Smith no consideraba la posibilidad de que un país tuviera la ventaja absoluta en la producción de todos los bienes, caso que podría darse entre países con diferentes niveles de desarrollo enfrentados en la competencia del mercado internacional. Por ello, plantea que si el costo del trabajo relativo de los bienes en cada país (no los costos 
absolutos) determina el valor en los intercambios internacionales, entonces los países de diferente tamaño podrían beneficiarse del comercio internacional, y denominó a esta condición ventaja comparativa.

Así, en los supuestos clásicos de Smith y Ricardo, todos los individuos ganan dentro del comercio, dado que la menor diferencia relativa de costos permite la especialización y genera intercambio comercial, basando su valor en un solo factor de producción: el trabajo. Por el contrario, la teoría neoclásica del comercio internacional considera que los patrones del comercio no dependen de la productividad de la mano de obra sino de la interrelación entre la cantidad de factor productivo que tiene un país y la cantidad de factor productivo que requiere un bien para su producción.

Su principal exponente es Bertil Ohlin, quien parte del teorema de su profesor Eli Heckscher, y construye un modelo tomando en consideración que los factores de producción necesarios para la elaboración de un bien son el trabajo y el capital, y asume que los países desarrollados tienen abundancia de capital mientras que los países en desarrollo tienen abundancia de trabajo. De esta manera, los postulados del modelo Heckscher-Ohlin, plantean que aquellos bienes que requieren mayor cantidad de trabajo para su producción deben ser producidos por los países con abundancia en mano de obra, mientras que los bienes que requieren capital de forma intensiva en su creación deben ser producidos por los países con abundancia en capital. El comercio internacional se basaría, entonces, en la interacción entre la intensidad y la abundancia factorial.

Sin embargo, aunque estos modelos sentaron las bases teóricas del comercio internacional no podían explicar la diferencia en los resultados de exportación entre países con recursos similares, especialmente porque se basaban en supuestos de competencia perfecta. De esta manera, con la instauración del sistema monetario internacional, empiezan a surgir los primeros postulados teóricos que trataban de explicar estas diferencias bajo la óptica de la dinámica propia de la empresa y no respecto a los factores de producción de los países.

Para Hymer (1960), la internacionalización no se fundamenta solamente en una ventaja en costos de producción como planteaban los clásicos sino, además, en la búsqueda de oportunidades para intercambiar capitales e incrementar la rentabilidad, considerando que la movilidad de capitales podrá ser incentivada en aquellas empresas que encuentren, fuera de sus fronteras, mejores opciones de financiamiento e inversión más allá del análisis del interés, estructuras organizacionales ventajosas e incluso facilidades de mercadeo, gracias a las diferencias estructurales existentes entre los mercados.

De forma similar, Vernon (1966) enfoca su teoría en el modelo de ciclo de vida del producto y cuestiona los preceptos clásicos al considerar que la innovación, la incorporación de economías a escala y el efecto de la incertidumbre son factores prioritarios para comprender los procesos del comercio internacional y la internacionalización de las empresas:

El presente artículo se ocupa de una línea prometedora de generalización y síntesis que me parece que ha sido algo descuidada por la corriente principal de la teoría del comercio. Pone menos énfasis en la doctrina de costos comparativos y más en el momento de la innovación, los efectos de las economías de escala y el papel de la ignorancia y la incertidumbre en la influencia de los patrones comerciales. (Vernon, 1966, p. 191, [traducción de la autora]).

Así mismo, Williamson (1975), afirma que la internacionalización de las empresas dependerá de los costos de transacción presentes en los contratos de negociación que variarán en cada mercado. Para ello toma como base los estudios de Coase (1937), Barnard (1938), Hayek (1945), Chandler (1962) y Arrow (1962) quienes apuntan que la organización económica tiene como problema central la adaptación autónoma a los mercados y que dicha adaptación es deliberada al interior de las firmas (Aranda y Montoya, 2006). 
Kindleberger (1969) y Hymer (1976) proponen la Teoría de la Organización Industrial, según la cual la internacionalización de la producción tendrá lugar cuando las empresas puedan desarrollar una ventaja competitiva exclusiva (monopolística) dentro de su proceso de producción, bien sea por características de tecnología, diversificación, comercialización, entre otras, que pueda ser transferible a las inversiones externas.

En otras palabras, la diferencia en la dotación factorial de la economía clásica y neoclásica sólo daría lugar a la especialización interindustrial, es decir, al intercambio entre diferentes industrias sobre las cuales se establecen las ventajas de cada país; por lo tanto, factores como las economías de escala, las imperfecciones de mercado, la diferenciación del producto y los gustos de los consumidores no estarían incorporadas dentro de las razones que fomentan el intercambio comercial y la internacionalización de las empresas. Estas falencias fueron resaltadas por Grubell y Lloyd (1975) quienes, a través de la definición del Comercio Intraindustrial, explicaron que el intercambio de las mismas industrias sería mayor entre los países con estructuras económicas y proporciones factoriales similares, lo que fomentaría la internacionalización.

Este sería el argumento con el que Fujita y Krugman (2004) explicarían que las concentraciones empresariales (clústers) se estaban generando como consecuencia de la nueva geografía económica, formando sistemas complejos que interrelacionaban a las empresas y a las industrias a través de la concentración geográfica de la producción, como la estructura centro-periferia de la economía global.

De esta manera, según Cardozo, Chavarro y Ramírez (2007:6) "a finales de los años setenta, la internacionalización se establece como una nueva línea de investigación, constituyéndose en la contribución más importante de los microeconomistas al conocimiento de la empresa multinacional”.

Esta contribución puede evidenciarse dentro de los aportes de Bucley y Casson (1976), quienes afirman, en su Teoría de Internacionalización, que las organizaciones multinacionales pueden servir como mecanismos alternativos para generar valor fuera de las fronteras nacionales cuando puedan eludir los costos de la imperfección de los mercados, siempre y cuando se cumplan dos condiciones: primera, ventajas al localizar la actividad en el exterior y, segunda, ejecutar actividades que sean más eficientes que la venta o cesión de la organización (Cardozo et. al, 2007).

Una síntesis de las contribuciones anteriores se puede observar en el Paradigma Ecléctico de Dunning (1977), que considera dos tipos de inversión. Una referida a las actividades económicas relacionadas con la exportación de bienes y servicios pero que se desarrollan dentro de las fronteras nacionales (comercio tradicional) y otra con la búsqueda de recursos, por parte de los agentes económicos, para producir bienes y servicios que puedan abastecer los mercados extranjeros (inversión extranjera). Según este modelo:

El incentivo para internacionalizar se deriva de la existencia de imperfecciones del mercado. Pueden ser estructurales, como p. Ej. barreras a la competencia, o cognitivas, p. ej. conocimiento imperfecto. Como se mencionó anteriormente, el paradigma ecléctico es una síntesis de otros enfoques que se concentran en el comercio o la producción internacional, en la posesión de tecnología superior o en estructuras de mercado imperfectas. (Carrizo, 2009, p. 51, [traducción de la autora]).

Ahora, las teorías anteriores parten de los postulados de las teorías de ubicación del producto, esbozadas por Hymer (1960) y Vernon (1966), enfatizando que la internacionalización es un proceso secundario que se basa en la creación de un producto que primero debe satisfacer las necesidades del mercado local, por lo que la exportación tendría mayor impacto dentro de las economías similares. Sin embargo, esto conlleva a la estandarización del producto y a una percepción de que la inversión extranjera podría resultar en un proceso de sustitución de importaciones.

Por ello, Kojima (1982) considera que el mercado puede generar oportunidades para una inversión que puede disminuir bienestar al desplazar comercio (desviación), como aseguran los planteamientos del ciclo de vida del 
producto y, además, una inversión capaz de reestructurar la industria del país con base en una ventaja comparativa dinámica que transforme las plataformas de exportación (creación). Acorde con Carrizo (2009), el argumento de Kojima resulta pertinente especialmente para las economías emergentes que pueden apuntar a invertir dentro de sus fronteras para aumentar producción, y fuera de ellas para expandir sus inversiones.

Finalmente, adicional a los aportes anteriores, Porter (1990) señala que una nación podrá generar ventajas competitivas en la medida en que pueda generar productividad, es decir, cuando pueda aumentar el valor de la producción por unidad de trabajo o capital; y no sólo cuando se toman en consideración los factores macroeconómicos, la distribución y los costos de la mano de obra, la abundancia de recursos naturales, las políticas públicas proteccionistas, o las prácticas administrativas o gerenciales (Porter, 1990).

En suma, desde la formalización de las teorías del comercio internacional bajo la perspectiva económica, son los costos de producción y las imperfecciones del mercado las principales variables de estudio para explicar no sólo la expansión internacional de las empresas sino también la dinámica de aprendizaje que se da al interior de estas.

Discusión: El componente epistemológico de las teorías de internacionalización

Según Padrón (2007, 2014), para sistematizar los enfoques epistemológicos se utilizan dos variables: gnoseológica, referida a las convicciones acerca de la fuente del conocimiento (empirismo o racionalismo), y ontológica, referida a las convicciones acerca de las relaciones del sujeto con la realidad (idealismo o realismo).

\begin{tabular}{|c|c|c|}
\hline $\begin{array}{c}\text { VARIABLE GNOSEOLÓGICA } \rightarrow \text { VARIABLE } \\
\text { ONTOLÓGICA } \downarrow\end{array}$ & EMPIRISMO & RACIONALISMO \\
\hline IDEALISMO & $\begin{array}{c}\text { Vivencialista - Experiencialista } \\
\text { Etnografía, diseños de convivencia, } \\
\text { inducción reflexiva... }\end{array}$ & $\begin{array}{c}\text { Vivencialista - Interpretativista } \\
\text { Interpretaciones libres, lenguajes } \\
\text { amplios, argumentación reflexiva... }\end{array}$ \\
\hline REALISMO & $\begin{array}{l}\text { Empirista - Inductivista } \\
\text { Mediciones, experimentaciones, } \\
\text { inducción controlada... }\end{array}$ & $\begin{array}{c}\text { Racionalista - Deductivista } \\
\text { Abstracciones, sistemas lógico- } \\
\text { matemáticos, deducción controlada... }\end{array}$ \\
\hline
\end{tabular}

De estas combinaciones se pueden obtener una serie de paradigmas como el positivismo (empirista inductivista), la hermenéutica (vivencialista - interpretativista), la fenomenología (vivencialista experiencialista) o el constructivismo (vivencialista - experiencialista); cada una con sus propios principios y postulados. Así, de acuerdo con la evolución teórica de la economía internacional descrita previamente, los diversos constructos creados para explicar el porqué de la internacionalización de las empresas forjan sus bases en estos paradigmas.

\section{Positivismo}

Augusto Comte, filósofo francés y padre del positivismo, afirmó dentro de la advertencia de su obra Curso de Filosofía Positiva:

Empleo la palabra filosofía en la acepción que le daban los antiguos, i particularmente a Aristóteles, para los cuales esa palabra designa el sistema jeneral de las concepciones humanas; $y$, añadiendo la palabra positiva, anuncio que considero que esta materia especial de filosofía que consiste en considerar que las teorías, en cualquier orden de ideas que sea, tienen por objeto únicamente la coordinación de los hechos observados, es lo que constituye el tercero i último estado de la filosofía general. (Comte, 1875, p. 67) 
En este contexto, las teorías clásicas y neoclásicas del comercio internacional, representadas por Adam Smith, David Ricardo y el modelo Heckscher-Ohlin, parten del paradigma positivista al considerar que la observación realizada sobre la dinámica del intercambio podía ser expresada en términos matemáticos a través de las ecuaciones de los precios relativos, basados en una serie de supuestos que determinaban las características de los factores de producción.

Así, dado que el positivismo es una aproximación epistemológica que combina el racionalismo con el empirismo y relaciona la lógica deductiva e inductiva, centrado en los paradigmas de las ciencias naturales que se basan en métodos cuantitativos, las primeras teorías del comercio internacional pueden ser consideradas altamente positivistas dado que se enfocaban en la explicación causal y la predicción del intercambio comercial.

De la mano estarían las teorías desarrolladas por Hymer (1960), quien hace una aproximación teórica con base en un método cuantitativo al definir como variables explicativas de la internacionalización a la rentabilidad, la tasa de interés y el mercadeo; Williamson (1975), quien lo hace considerando el costo de transacción de la negociación, pero mantiene el enfoque hipotético-deductivo; o Kindleberger (1969) quien considera a la ventaja competitiva monopolística como variable determinante de su teoría.

Entonces, dentro de estas teorías de internacionalización prevalece el hecho sobre la idea, dado que la comprobación de los postulados daba validez al conocimiento y, además, se generalizaba como pensamiento principal de la economía internacional. No se busca la razón por las cuales se generan las imperfecciones del mercado, solo se toma como hecho que existen y se buscan mejores escenarios con base en la medición de las variables de interés definidas.

\section{Interpretativismo}

Como contraposición al paradigma positivista, el interpretativismo es una perspectiva de investigación cualitativa que se basa en la comprensión de los hechos con base en el rol de los actores sociales. Para Weber (1922, p.6):

Toda interpretación, como toda ciencia en general, tiende a la "evidencia". La evidencia de la comprensión puede ser de carácter racional (y entonces, bien lógica, bien matemática) o de carácter endopático: afectiva, receptivo-artística. En el dominio de la acción es racionalmente evidente, ante todo, lo que de su "conexión de sentido" se comprende intelectualmente de un modo diáfano y exhaustivo. Y hay evidencia endopática de la acción cuando se revive plenamente la "conexión de sentimientos" que se vivió en ella.

En este sentido, según la técnica hermenéutica, basada en la interpretación de un texto cuya comprensión no sea evidente, no podría afirmarse una interpretación como única porque esta interpretación implica que "quien quiere lograr la comprensión de un texto tiene que desplegar una actitud receptiva dispuesta a dejarse decir algo por el argumento" (Arráez, Calles y Moreno, 2006, p. 176).

Schleiermacher (1768-1834) estableció que la interpretación debía seguir una serie de principios contextuales y psicológicos para alcanzar el mayor nivel de comprensión: los primeros basados en la gramática, pues esta permite descifrar el significado de una palabra con base en el contexto lingüístico dentro del cual se encuentre; mientras que los segundos estarían basados en el pensamiento del autor, dado que sólo cuando se reviven las experiencias del autor se puede llegar a comprender completamente el texto (Arráez, Calles y Moreno, 2006).

De forma contradictoria, para Dilthey, alumno de Schleiermacher, la experiencia no puede ser percibida, sino que se genera un acto reflexivo de conciencia, por lo que la experiencia representaría la vida. En este sentido, como lo reflejan Da Trinidade y López (2015, p.335): "Dilthey define la experiencia o "experiencia vivida" como una unidad cuyos elementos permanecen unidos por un significado común". 
Ahora, el debate se centraría en discutir que, como lo planteó Nietzsche, no hay hechos sino interpretaciones. De acuerdo con Catalán y Zabala (2011, p.27), los hechos no son tan independientes como se quisiera pensar, especialmente cuando se toma en cuenta que:

La ciencia no piensa porque no lo necesita, sino que más bien calcula dentro de ciertos paradigmas. Por supuesto que no hay nada de malo en ello, pero su resultado vale para su mismo paradigma, no para el pensamiento en general.

Bajo este enfoque tendrían cabida las teorías expuestas por Vernon (1966) y Grubell y Lloyd (1975), pues dentro de estas motivaciones teóricas se incorporan factores que dependerán de la interpretación de cada individuo. En el caso de Vernon, en su modelo de ciclo de vida del producto se toma como condicionante la incertidumbre y la ignorancia que tengan las organizaciones sobre las tendencias comerciales, factor netamente dependiente de la interpretación de cada sujeto (empresa). Mientras que, en el caso del comercio intraindustrial, planteado por Grubell y Lloyd, los gustos de los consumidores son determinantes para explicar el intercambio entre países, factor que implica que la experiencia no podrá ser percibida sino será producto de la reflexión consciente de los individuos (consumidores) acorde con su experiencia de vida.

De esta manera, estas dos teorías de internacionalización basan sus paradigmas en la interpretación y comprensión de los hechos acorde con las experiencias propias de los agentes del mercado, bien sea productores o consumidores. En este orden de ideas, tal como lo planteaba Kuhn, la innovación científica no proviene de la acumulación del conocimiento sino de una aproximación a la verdad que puede cambiar en la medida en que cambie el paradigma. Por ello, la insuficiencia de directrices metodológicas implicará que diferentes individuos puedan llegar a conclusiones particulares dependientes de su experiencia anterior (Kuhn, 1962). Es decir, los criterios epistemológicos de la economía internacional no son estáticos, sino que se transforman en la medida en que se desarrolla: si no se toman en cuenta las dinámicas propias de las ciencias sociales, las discrepancias frente a los posibles resultados de un ejercicio científico harán imposible el progreso en la ciencia.

En este punto, es valioso el aporte de Gadamer, quien pretende integrar el progreso de la ciencia con el progreso del pensamiento dentro de una sola concepción de la experiencia que se fundamente en un lenguaje común, implicando así la comprensión de la solidaridad humana (Aguilar, 2004). Sin embargo, este pensamiento basado en el lenguaje común no se evidencia aún dentro de las teorías de la economía internacional dado que, como problemática propia de la ciencia económica, se persigue un interés individual en la dinámica de los objetos de estudio. Así, cuando el método pretende igualar todo, se pierde la comprensión que se origina en la búsqueda de contradicciones e inconsistencias, y las interpretaciones dependerán de lo que se considere irracional.

Ahora bien, es dentro del análisis de este método invariante en donde tiene cabida la filosofía fenomenológica. Su fundador, Edmund Husserl, partía del principio de que la ciencia había dejado de ser significativa para el hombre porque carecía de criterios rigurosos para guiar la conducta de la humanidad, por lo que la fenomenología pretende fundamentar la objetividad del saber a través del uso de un método que permita mostrar un ente en sentido propio (sin apariencia), es decir, un fenómeno (León, 2012). Es con esta base que, posteriormente, Heidegger manifiesta que la fenomenología estaría enfocada en la interpretación de la estructura fundamental de la experiencia vivida.

Empero, bajo la postura más pragmática del pensamiento: si el lenguaje que acompaña al método es universal, se producirá la misma reacción en todos los individuos dada la reacción a ciertos símbolos. Esto se traduce en que el discurso lógico, que está implícito dentro del lenguaje, está directamente relacionado con el dominio de la interacción social. De esta manera, dentro del interaccionismo simbólico la objetividad social es ajena. (Carabaña y Lamo, 1978) 
Esta interacción social, dentro de la cual la estructura de la experiencia vivida es la base del conocimiento, se puede evidenciar en la teoría de internacionalización de Bucley y Casson (1976), dado que al considerar a las organizaciones como mecanismos de valor parten de un lenguaje universal que considera al valor como discurso base para la interacción social.

\section{Constructivismo}

Otro paradigma presente dentro de las teorías de internacionalización es el constructivismo, que hace referencia a la formación del conocimiento que se origina desde el interior del sujeto, quien construye el conocimiento desde la realidad a través de los mecanismos cognitivos de los que dispone. Lo anterior implica que la construcción estará relacionada con las situaciones que experimenten tanto el sujeto como el objeto, haciendo que ambos sean proclives a transformaciones. Así, para Araya, Alfaro y Andonegui (2007, p.77), el constructivismo se traduce en una "superación del antagonismo entre posiciones racionalistas y empiristas". En este sentido, la célebre frase de Descartes "pienso, luego existo" señala la separación clara entre lo mecánico y lo existencial.

Su principal exponente fue Kant, que en el siglo XVII propuso un compromiso entre racionalismo y empirismo, pues el primero exagera el papel del sujeto mientras que el segundo lo menosprecia. Posteriormente, Piaget analizó al aprendizaje como un proceso interno de construcción en el que el individuo participa activamente en la medida en que adquiere estructuras más complejas de pensamiento, que: evolucionan a partir de reflejos innatos, se organizan en esquemas de conducta, se internalizan en modelos de pensamiento y se desarrollan en estructuras individuales complejas.

El constructivismo es un modelo con más de un planteamiento, es una propuesta cognitiva que a través de varios enfoques (complementarios o contrapuestos) propone que el conocimiento no es una copia de lo real: "a partir de la acción sobre los objetos y con los otros sujetos, se efectúan combinaciones de relaciones que permiten aprender inteligentemente y tomar conciencia de las operaciones" (Londoño, 2008, p.76).

En este sentido, de acuerdo con Pérez (2012), el constructivismo se aproxima a la creación de sentido del sujeto cuando interactúa con su entorno sociocultural, sin embargo, este sentido puede enfocarse en un carácter totalmente social del lenguaje en el que los conocimientos se generan por una relación social de los individuos (constructivismo relativista) o por la influencia del poder de la actividad humana sobre la realidad social (constructivismo crítico).

Bajo este contexto, se puede encontrar el aprendizaje inteligente dentro del argumento de Fujita y Krugman (2004), quienes, al plantear la internacionalización con base en la nueva geografía económica, señalan como un clúster se generan producto del aprendizaje empresarial en donde cada sujeto (empresas) participa dentro de un sistema cada vez más completo a través de la creación de un conocimiento fomentado por la relación social de los individuos.

Similar sería el contexto epistemológico del paradigma ecléctico de Dunnin (1977), donde el constructivismo crítico es el fundamento detrás de la búsqueda de los nuevos recursos por parte de las empresas o, en otras palabras, la creación de sentido del sujeto (empresa) se da en la búsqueda de las inversiones eficientes en el extranjero. Sucede lo mismo dentro de la hipótesis de Kojima (1982) y Porter (1990), tomando en consideración que sus argumentos se basan en el poder de transformación interna de las empresas mientras interactúan con el mercado, creando cambios (procesos de aprendizaje) tanto internos como externos.

Así, como punto focal del constructivismo presente dentro de las teorías de la internacionalización, el primer concepto de un orden racional se deriva del modelo estándar de la ciencia socioeconómica, denominado como racionalidad constructivista por Hayek, quien consideraba que el elemento teórico de la economía: 
"Conduce hacia modelos racionales de predicción de la elección que generan hipótesis de investigación que se han estado probando en el laboratorio desde mediados del siglo XX. Aunque los resultados de estas pruebas suelen confirmar dicha hipótesis en las transacciones impersonales de mercado, es bien sabido que los resultados son recalcitrantemente ambiguos en el caso de las transacciones personales". (Smith, 2005:201)

\section{Conclusiones}

La economía internacional busca explicar el comportamiento de los intercambios comerciales entre los países, así como las interacciones financieras y los estímulos que puedan tener las economías para integrarse en búsqueda de un beneficio mayor. Para ello, las teorías de la internacionalización se basan en una serie de postulados macro y microeconómicos cuyo soporte principal se da en el fundamento matemático, para identificar las leyes que explican la realidad. Así, los argumentos teóricos clásicos y neoclásicos parten de modelos que infieren comportamientos para explicar las interacciones entre los países a través del estudio de variables como el costo de producción o la dotación factorial de las economías, definidos por esquemas con argumentos lógicos, aunque reduccionistas.

Sin embargo, a pesar de la importancia que el positivismo tiene dentro del pensamiento económico, la generalización propia del método científico no puede ser aplicada para todo el análisis de la internacionalización empresarial dado que los agentes que la conforman tienen intereses y condiciones diferentes que implican un impacto disímil ante cada política planteada. Por ende, no todas las leyes, principios y supuestos matemáticos pueden sostenerse para todos los fenómenos; por ende, la base positivista con la que se estructura la economía -como teoría- no puede responder a todas las dinámicas de la internacionalización.

En este orden de ideas, también existe espacio para la interpretación y la construcción dentro de la generación de constructos en la economía internacional. Por un lado, podría considerarse que la creación de valor como argumento básico de la internacionalización empresarial demuestra que el interpretativismo puede identificarse dentro de los mecanismos de aprendizaje de las organizaciones. Por otro lado, también se puede observar que el constructivismo está presente en los enfoques teóricos que perciben las relaciones sociales de los individuos.

De esta manera, existe una característica que se puede evidenciar en la evolución de las teorías que estudian a la internacionalización: con el tiempo demuestran transiciones entre los argumentos positivistas, interpretativistas y constructivistas; que incluso podrían señalarse como un ciclo acorde con la evolución del estudio epistemológico per se. Por lo anterior, también vale la pena resaltar que la cantidad de factores que componen el estudio de la internacionalización empresarial son tantos que los diferentes enfoques epistemológicos no necesariamente deben ser excluyentes, por lo que la explicación de la expansión organizacional puede tomar diferentes vertientes para su comprensión ${ }^{2}$.

\section{Referencias bibliográficas}

Aguilar, L. 2004. La hermenéutica filosófica de Gadamer. Revista Electrónica Sinéctica, 24: 61-64. http://www.redalyc.org/pdf/998/99815918009.pdf

\footnotetext{
${ }^{2}$ Incluyendo los otros que no son objeto de estudio en este análisis pero que podrían considerarse en siguientes investigaciones.
} 
Aranda, Y. V.; Montoya, I.A. 2006. Principales enfoques conceptuales explicativos del proceso de internacionalización de empresas. Revista Agronomía Colombiana, 24(1): 170-181. http://www.scielo.org.co/pdf/agc/v24n1/v24n1a19.pdf

Araya, V.; Alfaro, M.; Andonegui, M. 2007. Constructivismo: orígenes y perspectivas. Revista de Educación Laurus, 13(24): 76-92. http://www.redalyc.org/articulo.oa?id=76111485004.pdf

Arráez, M., Calles, J., Moreno, L. 2006. La Hermenéutica: una actividad interpretativa. Revista Sapiens. Revista Universitaria de Investigación, 7(2):171-181. https://www.redalyc.org/pdf/410/41070212.pdf

Borgucci, E. y Castellano, A. 2016. La teoría económica y la epistemología. Económicas CUC, 37(1): 9-40. DOI: http://dx.doi.org/10.17981/econcuc.15.5.2016.01

Carabaña M., J.; Lamo, E. 1978. La teoría social del interaccionismo simbólico. Revista española de investigaciones sociológicas, 1: 159-204. https://dialnet.unirioja.es/servlet/articulo?codigo=666889

Cardozo, P.P.; Chavarro, A.; Ramirez, C.A. 2007. Teorías de la Internacionalización. Revista Panorama, 1(3): 4-23. https://dialnet.unirioja.es/servlet/articulo?codigo $=4780130$

Carrizo M., A. 2009. The evolution of internationalization: towards a new theory?. Economia Global e Gestão. 14(1): 41-59. http://www.scielo.mec.pt/pdf/egg/v14n1/v14n1a04.pdf

Catalán, J.; Zabala, S. 2011. ¿Por qué no hay hechos sino sólo interpretaciones?. Revista Claves de Razón Práctica. 211: $\quad$ 26-29. https://static1.squarespace.com/static/549886d5e4b027d892383c5d/t/54fc53ade4bog1c87ea64cb2/14258226 37713/Catalan_Zabala+CLAVES.pdf

Comte, A. 1875. Principios de Filosofía Positiva. Traducción por Jorge Lagarrigue, 2012. Santiago: Imprenta de la Librería de Mercurio. http://www.cervantesvirtual.com/nd/ark:/59851/bmc3r144

Da Trinidade, H.; López C., Y. 2015. La Hermenéutica en el pensamiento de Whilhel Dilthey. GRIOT, Revista de Filosofía. 11(1). http://oaji.net/articles/2015/2742-1451076947.pdf

De la Hoz, P. 2013. Generalidades de Comercio Internacional. Medellín, Colombia: Centro Editorial Esumer. http://www.esumer.edu.co/images/centroeditorial/Libros/fei/libros/generalidadesdecomerciointernacional.p df

Fujita, M.; Krugman, P. 2004. The new economic geography: Past, present and the future. Papers Reg. Sci. 83:139164. DOI: 10.1007/s10110-003-0180-0

Gómez R., A. .2001. Las leyes ceteris paribus y la inexactitud de la economía. Revista Teorema, 20(3): 69-80. https://dialnet.unirioja.es/servlet/articulo?codigo $=4250281$

Grubel, M.; Lloyd, P. 1975. Intra-industry Trade: The theory of measurement of International Trade in Differentiated Products. The Macmillan Press LTD.

Hymer, S. 1960. The international operations of national firms, a study of direct foreign investment. Thesis (Ph. D.) Massachusetts Institute of Technology, Dept. of Economics. https://dspace.mit.edu/handle/1721.1/27375

Kojima, K. 1982. Macroeconomic versus International Business: approach to Direct Foreign Investment. Hitotsubashi Journal of Economics. 3 (1): 1-19. DOI: $10.15057 / 7932$. 
Kuhn, T. 1962. La Estructura de las Revoluciones Científicas. Octava reimpresión, 2004. Fondo de Cultura Económica: Argentina. https://www.perio.unlp.edu.ar/catedras/system/files/kuhn_thomas _la_estructura_de_las_revoluciones_cientificas_o.doc

León, E. 2012. El giro hermenéutico de la fenomenología en Martín Heidegger. Revista Polis, 22: 1-14. https://journals.openedition.org/polis/2690\#text

Londoño R., C. 2008. Avatares del constructivismo: de Kant a Piage. Revista Historia de la Educación Latinoamericana, 10: 73-96. http://www.redalyc.org/pdf/869/86901006.pdf

Padrón, J. 2007. Tendencias Epistemológicas de la Investigación Científica en el Siglo XXI. Cinta de Moebio, 28:128. www.moebio.uchile.cl/28/padron.html

Padrón, J. 2014. ¿Qué es la epistemología?. Epistemología en DVD. http://padron.entretemas.com.ve/QueEsLaEpistemologia.pdf

Pérez R., A. 2012. Sobre el Constructivismo: construcción social de lo real y práctica investigativa. Revista Latinoamericana de Metodología de las Ciencias Sociales, 2(2): 5-21. https://www.relmecs.fahce.unlp.edu.ar/article/download/RELMECSv02noza02/pdf/

Porter, M. 1990. The competitive advantage of nations. Harvard Business Report, 90(2): 73-91. https://hbr.org/1990/03/the-competitive-advantage-of-nations

Ramos B., M.G.; Bello, S. (En proceso de diseño, 2020). Enseñanza de matemática en economía: el problema de la abstracción. En Formación y pensamiento crítico: reflexiones y métodos de enseñanza en economía. Capítulo de libro no publicado. Bogotá: Politécnico Grancolombiano.

Real Academia Española (2019). Diccionario de Lengua Española. Recuperado de: https://dle.rae.es/?id=9AwuYaT

Ricardo, D. 1817. Principios de Economía Política y Tributación. Traducción de Juan Broc B., Nelly Wolff y Julio Estrada M, 1959. México: Fondo de Cultura Económica.

Sastre, B. 2014. Evolución del Comercio Internacional: Consecuencias de la globalización (tesis de pregrado). Universidad de Valladolid, Valladolid, España. https://uvadoc.uva.es/bitstream/10324/7357/1/TFG-E-19.pdf

Smith, A. 1776. Investigación sobre la naturaleza y causas de la riqueza de las naciones. Traducción de Gabriel Franco. Décima reimpresión, 1999. México: Fondo de Cultura Económica.

Smith, V. 2005. Racionalidad constructivista y ecología en Economía. Revista Asturiana de Economía, 32: 197-273. http://www.revistaasturianadeeconomia.org/raepdf/32/vernonRae32.pdf

Vernon, R. 1966. International investment and international trade in the product cicle. Quarterly Journal of Economics, MIT Press. https://pdfs.semanticscholar.org/3935/67ef46dc9229d22234e3210d5d62005a6a8f.pdf

Weber, M. 1922. Economía y Sociedad. Cuarta edición en alemán, 1964. España: Fondo de Cultura Económica. https://zoonpolitikonmx.files.wordpress.com/2014/08/max-weber-economia-y-sociedad.pdf 